\title{
On accurate computations of slowly convergent atomic properties in few-electron ions and electron-electron correlations.
}

\author{
Alexei M. Frolov* \\ Department of Applied Mathematics \\ University of Western Ontario, London, Ontario N6H 5B\%, Canada \\ David M. Wardlaw $\dagger$ \\ Department of Chemistry, Memorial University of Newfoundland, \\ St.John's, Newfoundland and Labrador, A1C 5S7, Canada
}

(Dated: October 16, 2018)

\begin{abstract}
We discuss an approach to accurate numerical computations of slowly convergent properties in two-electron atoms/ions which include the negatively charged $\mathrm{Ps}^{-}\left(e^{-} e^{+} e^{-}\right)$and $\mathrm{H}^{-}$ions, $\mathrm{He}$ atom and positively charged, helium-like ions from $\mathrm{Li}^{+}$to $\mathrm{Ni}^{26+}$. All these ions are considered in their ground $1^{1} S$-state(s). The slowly convergent properties selected in this study include the electron-nulceus $\left\langle r_{e N}^{2 k}\right\rangle$ and electron-electron $\left\langle r_{e e}^{2 k}\right\rangle$ expectation values for $k=2,3,4$ and 5 .

PACS number(s): 36.10.-k and 36.10.Dr

First version 25.10.2015, Preprint-2015-15/1 (this is 3rd version, 7th of July 2016) [at.phys.], 17 pages.
\end{abstract}

${ }^{*} \mathrm{E}$-mail address: afrolov@uwo.ca

${ }^{\dagger} \mathrm{E}-\mathrm{mail}$ address: dwardlaw@mun.ca 


\section{INTRODUCTION}

In this communication we investigate the overall accuracy of representation of the exact wave functions by trial variational functions. This problem is of great interest in the physics of few-body systems, including few-electron atoms and ions. To formulate the problem let us consider a number of truly correlated two-electron atomic systems, i.e. two-electron atoms and ions. Recently, a large number of 'highly accurate', or even 'precise' methods have been developed to perform bound state computations in such systems. Almost all these methods can produce a very accurate (or essentially 'exact') numerical value of the total energy $E$ of some selected bound state. In addition to the total energy one also finds that highly accurate methods in applications to truly correlated systems allow one to determine a large number of bound state properties, or expectation values. Convergence rates of these expectation values vary between very fast and relatively slow, or even very slow. In general, for an arbitrary few-electron atom/ion one always finds a number of expectation values $\langle X\rangle$ which are difficult to determine to high and very high accuracy. The sources of such difficulties are mainly related to very slow convergence rates. For instance, for truly correlated atomic systems the expectation values of the $p_{e}^{2 n}, r_{e N}^{2 n}$ and $r_{e e}^{2 n}$ operators converge increasingly slowly as the power $2 n$ increases. For large $2 n$ powers, e.g., if $2 n \geq 10$, these expectation values converge very slowly. Note that this conclusion is true only for atomic systems for which contribution of the electron-electron correlations is sufficiently large. However, if the role of such correlations is small (negligible), then the expectation values of the $p_{e}^{2 n}, r_{e N}^{2 n}$ and $r_{e e}^{2 n}$ operators are simply related with the expectation values of the $p_{e}^{2}, r_{e N}^{2}$ and $r_{e e}^{2}$ operators, i.e. the problem can easily be solved by using such 'additional' relations. Below, we shall assume that the electron-electron correlations are relatively large for all systems mentioned in this study.

The goal of this study is to perform highly accurate computations of some slowly convergent expectation values $\left\langle r_{e N}^{2 n}\right\rangle$ and $\left\langle r_{e e}^{2 n}\right\rangle$ (where $2 n \geq 6$ ) for a large number of two-electron ions, including the negatively charged $\mathrm{Ps}^{-}$and $\mathrm{H}^{-}$ions, and a number of helium-like atoms and positively charged ions (all ions from $\mathrm{Li}^{+}$to $\mathrm{Ni}^{26+}$ ). Due to the electron-electron correlations mentioned above, these expectation values have never been calculated for such ions/atoms to high and very high accuracy. Therefore, our expectation values of the $r_{e N}^{2 n}$ and $r_{e e}^{2 n}$ operators can be considered as an important addition to the known bound state 
properties of all ions/atoms considered in this study. On the other hand, the knowledge of these expectation values allows one to understand the internal nature of electron-electron correlations and contribution of these correlations to slowly convergent bound state properties. In addition to these two factors, it should be mentioned that the electron-nucleus $\left\langle r_{e N}^{2 k}\right\rangle$ expectation value plays an important role in applcations to actual physical problems. Indeed, these expectation values are needed to determine electric multipole momenta of different orders in atoms and ions. The same values can be used to determine the form-factors of these atoms and ions, i.e. Fourier transformations $\exp (\imath \mathbf{q} \cdot \mathbf{r})$ of the one-electron density distribution function $\rho_{e}(\mathbf{r})$, or one-electron density, for short. The rigorous definition of the form-factor is (see, e.g., [1], [2]):

$$
F(\mathbf{q})=\int \exp (\imath \mathbf{q} \cdot \mathbf{r}) \rho_{e}(\mathbf{r}) d^{3} \mathbf{r}=\frac{4 \pi}{q} \int_{0}^{\infty} \sin (q r) \rho_{e}(r) r d r
$$

where we have assumed that the electron density $\rho_{e}(r)$ is spherically symmetric. For twoelectron ions with large electric charges $Q e$ this is a realistic and very accurate approximation. In addition, as follows from the general theory of bound state spectra the bound state wave function of atoms is always a real function, or it can be chosen as real. In the case of an ion/atom with $N_{e}$ bound electrons the atomic form-factor (of form-factor, for short) in Eq.(11) the non-Coulomb part in the last formula is reduced to the form

$$
f(q)=\left\langle r_{e N}^{2}\right\rangle-\frac{q^{2}\left\langle r_{e N}^{4}\right\rangle}{3 !}+\frac{q^{4}\left\langle r_{e N}^{6}\right\rangle}{5 !}-\frac{q^{6}\left\langle r_{e N}^{8}\right\rangle}{7 !}+\frac{q^{8}\left\langle r_{e N}^{10}\right\rangle}{9 !}-\frac{q^{10}\left\langle r_{e N}^{12}\right\rangle}{11 !}+\ldots
$$

where $r=r_{e N}$ is the electron-nucleus distance (scalar coordinate). In Eq.(22) we eliminated the factor $4 \pi$ which is usually compensated by the corresponding factors from the angular part of the total wave function. Here and everywhere below in this study we assume that the electron density $\rho_{e}(r)$ is normalized to the number of bound electrons $N_{e}$.

As follows from Eq.(2) numerical computations of form-factors for few-electrons atoms and ions are reduced to accurate calculations of a few $\left\langle r_{e N}^{2 k}\right\rangle$ expectation values ( $k=$ $1,2,3, \ldots)$. In turn, the form-factor determines cross-sections of many actual atomic processes and reactions, e.g., cross-sections of the elastic and non-elastic (electron) scattering, bremsstrahlung, positron annihilation, etc (see, e.g., [2], [3] and [4]). Numerous examples of calculations of different atomic cross-sections by using form-factors can be found in Quantum Electrodynamics (see, e.g., [5] and [6]). Formally, this means that, if we know a very few electron-nucleus $\left\langle r_{e N}^{2 k}\right\rangle$ expectation values, then we can evaluate a significant number of 
actual atomic properties. In other words, the electron-nucleus $\left\langle r_{e N}^{2 k}\right\rangle$ and electron-electron $\left\langle r_{e e}^{2 k}\right\rangle$ expectation values are important properties which are useful to know for an arbitrary bound state in few- and many-electron atoms and ions. However, by performing accurate atomic calculations it is easy to find that the both $\left\langle r_{e N}^{2 k}\right\rangle$ and $\left\langle r_{e e}^{2 k}\right\rangle$ expectation values become slowly convergent quantities already at $2 k=6$.

In this short communication we investigate the problem of slow convergence of these expectation values and determine the $\left\langle r_{e N}^{2 k}\right\rangle$ and $\left\langle r_{e e}^{2 k}\right\rangle$ expectation values (for $k=3,4,5$ ) for a large number of two-electron ions which include the neutral helium atom, the positively charged, helium-like $\mathrm{Li}^{+}-\mathrm{Ni}^{26+}$ ions $(3 \leq Q \leq 28)$ and the weakly-bound, negatively charged $\mathrm{Ps}^{-}$and $\mathrm{H}^{-}$ions, where the convergence of these expectation values is poor. For the last two ions we investigate convergence of these expectation values numerically by considering these values as a function of the total number of basis functions $N$. To simplify some parts of our analysis in this study we restrict ourselves to the case of the ground (bound) $1^{1} S$-state in each two-electron ion/atom considered. Another question discussed in this study is the explicit 'asymptotic' formulas for the $Q^{-1}$ expansion of these expectation values. After an extensive investigation of this probelm we have found that the existing $Q^{-1}$ expansion for the $\left\langle r_{e N}^{2 k}\right\rangle$ and $\left\langle r_{e e}^{2 k}\right\rangle$ expectation values (for $\left.k=3,4,5\right)$ needs to be modified. However, after relatively small modifications the $Q^{-1}$-expansion begins to work very well even for slowly convergent properties. Briefly, we can say that the accurate determination of the $\left\langle r_{e N}^{6}\right\rangle,\left\langle r_{e e}^{6}\right\rangle,\left\langle r_{e N}^{8}\right\rangle,\left\langle r_{e e}^{8}\right\rangle,\left\langle r_{e N}^{10}\right\rangle$ and $\left\langle r_{e e}^{10}\right\rangle$ expectation values in this paper can be considered as an important addition to the atomic properties known for each of the two-electron ions mentioned above.

\section{HAMILTONIAN AND WAVE FUNCTIONS}

The non-relativistic Hamiltonian $H$ of an arbitrary two-electron atom/ion takes the form (see, e.g., [4])

$$
H=-\frac{\hbar^{2}}{2 m_{e}}\left[\nabla_{1}^{2}+\nabla_{2}^{2}+\frac{m_{e}}{M} \nabla_{3}^{2}\right]-\frac{Q e^{2}}{r_{32}}-\frac{Q e^{2}}{r_{31}}+\frac{e^{2}}{r_{21}}
$$

where $\hbar=\frac{h}{2 \pi}$ is the reduced Planck constant and $m_{e}$ is the electron mass and $e$ is the absolute value of the electric charge of an electron. In this equation and everywhere below in this study the subscripts 1 and 2 designate two electrons $\left(e^{-}\right)$, while the subscript 3 denotes the 
positively charged atomic nucleus with the electric charge $Q e$. For all ions/atoms considered in this study we assume that the mass of the central atomic nucleus is infinite, i.e. $\frac{m_{e}}{M}=0$ in Eq.(3). For the $\mathrm{Ps}^{-}$ion the subscript 3 denotes the positron $\left(e^{+}\right)$with the mass $m_{e}$ (the same electron mass) and positive electric charge $+e$, or $e$. The mass ratio in Eq.(3) is $\frac{m_{e}}{M}=1$ for the $\mathrm{Ps}^{-}$ion. In fact, everywhere below, only atomic units $\hbar=1,|e|=1, m_{e}=1$ are employed. In these units the explicit form of the Hamiltonian H, Eq.(3), is simplified to the form

$$
H=-\frac{1}{2}\left[\nabla_{1}^{2}+\nabla_{2}^{2}+\frac{1}{M} \nabla_{3}^{2}\right]-\frac{Q}{r_{32}}-\frac{Q}{r_{31}}+\frac{1}{r_{21}}
$$

where the notations $r_{i j}=\left|\mathbf{r}_{i}-\mathbf{r}_{j}\right|=r_{j i}$ stand for three interparticle distances (= relative coordinates) which are the absolute values of differences of the Cartesian coordinates $\mathbf{r}_{i}$ of these three particles. Note that each relative coordinate $r_{i j}$ is a scalar which is rotationally and translationally invariant. However, these coordinates are not truly independent, since e.g., $\left|r_{32}-r_{31}\right| \leq r_{21} \leq r_{32}+r_{31},\left|r_{32}-r_{21}\right| \leq r_{31} \leq r_{32}+r_{21}$, etc. This produces a number of problems in computations of the three-particle integrals in the relative coordinates $r_{32}, r_{31}, r_{21}$. To simplify such calculations it is better to apply a set of three perimetric coordinates $u_{1}, u_{2}, u_{3}$ which are simply related to the relative coordinates: $u_{k}=\frac{1}{2}\left(r_{i k}+r_{j k}-\right.$ $r_{i j}$ ), while inverse relations take the form $r_{i j}=u_{i}+u_{j}$. The three perimetric coordinates $u_{1}, u_{2}, u_{3}$ are truly independent of each other and each of them varies between 0 and $+\infty$. The Jacobian of the transition $\left(r_{32}, r_{31}, r_{21}\right) \rightarrow\left(u_{1}, u_{2}, u_{3}\right)$ is a constant which equals 2 .

To solve the non-relativistic Schrödinger equation $H \Psi=E \Psi$ for the two-electron ions, where $E<0$, and obtain highly accurate wave function(s) we approximate the unknown exact solution of the non-relativistic Schrödinger equation by using some rapidly convergent variational expansions. The best of such expansions is the exponential variational expansion in the relative coordinates $r_{32}, r_{31}, r_{21}$, or in the perimetric coordinates $u_{1}, u_{2}, u_{3}$. For the ground (bound) $1^{1} S$-state of the two-electron ions/atoms the explicit form of this expansion is

$$
\begin{aligned}
\Psi & =\frac{1}{2}\left(1+\hat{P}_{12}\right) \sum_{i=1}^{N} C_{i} \exp \left(-\alpha_{i} r_{32}-\beta_{i} r_{31}-\gamma_{i} r_{21}\right) \\
& =\frac{1}{2}\left(1+\hat{P}_{12}\right) \sum_{i=1}^{N} C_{i} \exp \left[-\left(\alpha_{i}+\beta_{i}\right) u_{3}-\left(\alpha_{i}+\gamma_{i}\right) u_{2}-\left(\beta_{i}+\gamma_{i}\right) u_{3}\right]
\end{aligned}
$$

where the notation $\hat{P}_{12}$ stands for the permutation operator of identical particles (electrons), $C_{i}(i=1,2, \ldots, N)$ are the linear parameters of the exponential expansion, Eq.(15), while 
$\alpha_{i}, \beta_{i}$ and $\gamma_{i}$ are the non-linear parameters of this expansion. The non-linear parameters must be varied in calculations to increase the overall efficiency and accuracy of the method. The best-to-date optimization strategy for these non-linear parameters was described in [7], while its modified (advanced) version was presented in another paper published in 2006 (see the second paper in Ref.[7]). The $3 N$-conditions $\alpha_{i}+\beta_{i}>0, \alpha_{i}+\gamma_{i}>0, \beta_{i}+\gamma_{i}>0$ for $i=1,2, \ldots, N$ must be obeyed to guarantee convergence of all three-particle integrals which are needed in computations.

\section{CALCULATIONS OF THE EXPECTATION VALUES AND THREE- PARTICLE INTEGRLAS}

By using highly accurate, variational wave functions $\Psi$ constructed for the ground $1^{1} S$-state of the two-electron ions we can determine the expectation value of an arbitrary, in principle, self-adjoint operator $\hat{X}$ which can be defined for this system. This can be written in the following general form (see, e.g., [8])

$$
\langle\hat{X}\rangle=\frac{\langle\Psi \mid \hat{X} \Psi\rangle}{\langle\Psi \mid \Psi\rangle}
$$

where $\hat{X}$ is a self-adjoint operator which explicitly depends upon three relative coordinates $r_{32}, r_{31}$ and $r_{21}$. Formally, without loss of generality we can assume that our wave function has a unit norm, i.e. $\langle\Psi \mid \Psi\rangle=1$. Our interest in this study is restricted to the cases when in Eq.(6) we choose either $\hat{X}=r_{e N}^{2 k}$, or $\hat{X}=r_{e e}^{2 k}$, where $k=2,3,4$ and 5. At the beginning of this project we expected that such expectation values could be determined without any problem by using relatively short variational expansions, Eq.(6), which include, e.g., $N=700$ - 1000 basis functions. However, in actual computations we have found that such relatively short variational expansions of the wave functions allow one to determine only two/three correct decimal digits in each of the $\left\langle r_{e N}^{8}\right\rangle,\left\langle r_{e N}^{10}\right\rangle,\left\langle r_{e e}^{8}\right\rangle$, and $\left\langle r_{e e}^{10}\right\rangle$ expectation values. After a number of attempts to resolve this situation and improve the overall convergence rate for these expectation values we have decided to investigate this problem more carefully.

First, note that all matrix elements of the $r_{e N}^{2 k}$ and $r_{e e}^{2 k}$ operators in the exponential basis, Eq.(5), are written in the form of the corresponding Laplace transformations. The explicit expressions for these matrix elements in the relative coordinates take the form

$$
\mathcal{F}_{2 k+1 ; 1 ; 1}(\alpha, \beta, \gamma)=\int_{0}^{+\infty} \int_{0}^{+\infty} \int_{\left|r_{32}-r_{31}\right|}^{r_{32}+r_{31}} \exp \left[-\alpha r_{32}-\beta r_{31}-\gamma r_{21}\right] r_{32}^{2 k+1} r_{31} r_{21} d r_{32} d r_{31} d r_{21}
$$


where $k=2,3,4$ and 5 . These integrals belong to a special class of the general three-body integrals which are the Laplace transforms of the polynomial function $F\left(r_{32}, r_{31}, r_{21}\right)=$ $r_{32}^{k} r_{31}^{l} r_{21}^{n}$ of the three relative coordinates $r_{32}, r_{31}, r_{21}$. The general formula for such a threebody integral is written in the form

$$
\mathcal{F}_{k ; l ; n}(\alpha, \beta, \gamma)=\iiint r_{32}^{k} r_{31}^{l} r_{21}^{n} \exp \left(-\alpha r_{32}-\beta r_{31}-\gamma r_{21}\right) d r_{32} d r_{31} d r_{21}
$$

where all indexes $k, l, n$ are assumed to be non-negative. In perimetric coordinates the same integral, Eq.(17), is written in the form

$$
\begin{aligned}
\mathcal{F}_{k ; l ; n}(\alpha, \beta, \gamma)= & 2 \int_{0}^{+\infty} \int_{0}^{+\infty} \int_{0}^{+\infty}\left(u_{2}+u_{3}\right)^{k}\left(u_{1}+u_{3}\right)^{l}\left(u_{1}+u_{2}\right)^{n} \times \\
& \exp \left[-(\alpha+\beta) u_{3}-(\alpha+\gamma) u_{2}-(\beta+\gamma) u_{1}\right] d u_{1} d u_{2} d u_{3}
\end{aligned}
$$

Derivation of the closed analytical formula for the integral, Eq.(9), is straightforward in perimetric coordinates. The formula takes the from

$$
\begin{aligned}
& \mathcal{F}_{k ; l ; n}(\alpha, \beta, \gamma)=2 \sum_{k_{1}=0}^{k} \sum_{l_{1}=0}^{l} \sum_{n_{1}=0}^{n} C_{k_{1}}^{k} C_{l_{1}}^{l} C_{n_{1}}^{n} \frac{\left(l-l_{1}+k_{1}\right) !}{(\alpha+\beta)^{l-l_{1}+k_{1}+1}} \frac{\left(k-k_{1}+n_{1}\right) !}{(\alpha+\gamma)^{k-k_{1}+n_{1}+1}} \frac{\left(n-n_{1}+l_{1}\right) !}{(\beta+\gamma)^{n-n_{1}+l_{1}+1}} \\
= & 2 \cdot k ! \cdot l ! \cdot n ! \sum_{k_{1}=0}^{k} \sum_{l_{1}=0}^{l} \sum_{n_{1}=0}^{n} \frac{C_{n-n_{1}+k_{1}}^{k_{1}} C_{k-k_{1}+l_{1}}^{l_{1}} C_{l-l_{1}+n_{1}}^{n_{1}}}{(\alpha+\beta)^{l-l_{1}+k_{1}+1}(\alpha+\gamma)^{k-k_{1}+n_{1}+1}(\beta+\gamma)^{n-n_{1}+l_{1}+1}}
\end{aligned}
$$

where $C_{M}^{m}$ is the number of combinations from $M$ by $m$ (here $m$ and $M$ are integer nonnegative numbers). The formula, Eq.(10), can also be written in a few different (but equivalent!) forms. The formula, Eq.(10), was derived for the first time in the middle of 1980's [9]. The formula, Eq.(10), has been used in all calculations of the electron-nucleus $\left\langle r_{e N}^{2 k}\right\rangle$ and elecron-electron $\left\langle r_{e e}^{2 k}\right\rangle$ expectation values performed for this study.

\section{DISCUSSIONS AND CONCLUSION}

As mentioned above the $\left\langle r_{e N}^{2 k}\right\rangle$ and $\left\langle r_{e e}^{2 k}\right\rangle$ expectation values for $k=2,3,4$ and 5 have never been determined to high accuracy for almost all two-electron ions/atoms discussed in this study. This means that our results from Tables I - V can be considered as an important addition to the bound state properties known for such ions obtained in earlier studies (see, e.g, [10] for the $\mathrm{Ps}^{-}$ion, [11] for the $\mathrm{H}^{-}$ion and [12] for the positively charged, two-electron ions). In general, many of these expectation values for the $\mathrm{Ps}^{-}$and $\mathrm{H}^{-}$ions have been predicted numerically since the middle of 1980's (see, e.g., [14] and [15] for the Ps ${ }^{-}$ion). An 
analogous situation existed for the helium atom, and for some helium-like, positively charged ions. The goal of this study was to improve the accuracy of numerical predictions of these expectation values. Unfortunately, this simple approach to such a comparison of existing calculations to our new calculations does not work, since for the $\left\langle r_{e N}^{2 k}\right\rangle$ and $\left\langle r_{e e}^{2 k}\right\rangle$ expectation values we cannot compare our numerical values with the results from earlier studies (some comparison can be made only with the quantities obtained at the Hartree-Fock and lowest post-HF level). Therefore, in order to demostrate the current level of accuracy in this study we have developed and applied a few different tests. The most interesting test is the convergence investigation performed for the $\left\langle r_{e N}^{2 k}\right\rangle$ and $\left\langle r_{e e}^{2 k}\right\rangle$ expectation values. Convergence of the $\left\langle r_{e N}^{2 k}\right\rangle$ and $\left\langle r_{e e}^{2 k}\right\rangle$ expectation values with the total number of basis functions $N$ used in actual highly accurate computations in Eq.(15) is shown in Tables I (for $k=2$ and 3) and in Table II (for $k=4$ and 5) for the $\mathrm{Ps}^{-}$ion. Analogous results for the ${ }^{\infty} \mathrm{H}^{-}$ion look very similar (see Table III). In general, by comparing the expectation values from Tables I, II and III, computed with different numbers of basis functions $N$, we can approximately predict the total number of stable decimal digits in the final results.

Tables IV and V contain numerical values of the $\left\langle r_{e N}^{2 k}\right\rangle$ and $\left\langle r_{e e}^{2 k}\right\rangle$ expectation values in a.u. (for $k=3,4$ and 5 ) determined for a large number of two-electron ions in their ground $1^{1} S$-state(s). As follows from these Tables the actual $Q$-dependence of these expectaion values is smooth and can be investigated in detail. In particular, by using the $\left\langle r_{e N}^{2 k}\right\rangle$ and $\left\langle r_{e e}^{2 k}\right\rangle$ expectation values from Tables IV - V we can construct accurate asymptotic formulas for the $Q^{-1}$-expansion. The general ideology of the universal method of the $Q^{-1}$-expansion for few-electron atoms/ions is discussed in [13] and [8]. For our expectation values this 'direct' approach does not work well, since the both $\left\langle r_{e N}^{2 k}\right\rangle$ and $\left\langle r_{e e}^{2 k}\right\rangle$ expectation values vary with $Q$ in a very wide numerical range. Therefore, for these expectation values the classical method of the $Q^{-1}$-expansion must be modified in the following way. Instead of the $\left\langle r_{e N}^{2 k}\right\rangle$ and $\left\langle r_{e e}^{2 k}\right\rangle$ expectation values we have to use the logarithms of these values, i.e. $\ln \left\langle r_{e N}^{2 k}\right\rangle$ and $\ln \left\langle r_{e e}^{2 k}\right\rangle$ values which vary between the lower and upper boundaries, when $Q$ changes from unity up to 30 - 40. In reality, these two boundaries are very close to each other.

For these logarithms we can write the regular $Q^{-1}$-expansions, which starts from the first $Q^{2}$-term

$$
\ln \left\langle r_{e N}^{2 k}\right\rangle=a_{2} Q^{2}+a_{1} Q+a_{0}+b_{1} Q^{-1}+b_{2} Q^{-2}+b_{3} Q^{-3}+b_{4} Q^{-4}+\ldots
$$




$$
\ln \left\langle r_{e e}^{2 k}\right\rangle=A_{2} Q^{2}+A_{1} Q+A_{0}+B_{1} Q^{-1}+B_{2} Q^{-2}+B_{3} Q^{-3}+B_{4} Q^{-4}+\ldots
$$

This structure of the $Q^{-1}$-expansions follows from the explicit form of the Hamiltonian Eq.(44) which describes all two-electron ions/atoms considered in this study. The unknown coefficients in the right-hand sides of the equalities Eqs.(11) and (12) can be obtained by solving a system of linear equations with the known $\ln \left\langle r_{e N}^{2 k}\right\rangle$ and $\ln \left\langle r_{e e}^{2 k}\right\rangle$ expectation values. In general, the total number of unknown coefficients, e.g., $a_{2}, a_{1}, a_{0}, b_{1}, b_{2}, b_{3}, \ldots$ in Eq.(11), is always smaller than the total number of the computed expectation values $\ln \left\langle r_{e N}^{2 k}\right\rangle$ and $\ln \left\langle r_{e e}^{2 k}\right\rangle$. This means that we have to apply the method of least squares (see, e.g., [16]). After a number of trials we have obtained the numerical values of the unknown coefficients in Eqs.(11) and (12) (for $k=3,4$ and 5). These coefficients can be used in actual evaluations for different two-electron atoms/ions. Applications of the formula, Eq.(11), to determine the $\left\langle r_{e N}^{2 k}\right\rangle$ expectation values (for $2 k=6$ and 8 ) are illustrated by the results from Table VI. The goal of a series of calculations was to approximate the 'unknown' $\left\langle r_{e N}^{6}\right\rangle$ and $\left\langle r_{e N}^{8}\right\rangle$ expectation values for the $\mathrm{K}^{17+}$ ion and compare these quantities with the 'exact' numerical values obtained in a series of sepate (direct) calculations (see Table IV). Results from Table VI illustrate convergence of intermediate results to the 'exact' expectation values when the total number of terms $M$ in the $Q^{-1}$-expansion, Eq.(11), increases. In Table VI we consider expectation values obtanined for $M=14,16,18,20,22$ and 24 .

We have determined the $\left\langle r_{e N}^{2 k}\right\rangle$ and $\left\langle r_{e e}^{2 k}\right\rangle$ expectation values $(k=2,3,4$ and 5$)$ for the ground (bound) $1^{1} S$-state(s) in a large number of two-electron ions. These ions include the negatively charged, weakly-bound $\mathrm{Ps}^{-}$and $\mathrm{H}^{-}$ions, He atom and positively charged helium-like ions. Note that most of these expectation values have never been evaluated to high accuracy in earlier studies performed for these ions. Investigation of the $\left\langle r_{e N}^{2 k}\right\rangle$ and $\left\langle r_{e e}^{2 k}\right\rangle$ expectation values ( $k=2,3,4$ and 5$)$ allows us to understand convergence of these 'slowly convergent' expectation values upon the total number of basis functions $N$ used in calculations. Furthermore, the knowledge of the $\left\langle r_{e N}^{2 k}\right\rangle$ and $\left\langle r_{e e}^{2 k}\right\rangle$ expectation values can be used to predict the atomic form-factor and many other properties of atomic systems considered in this study. In general, the $\left\langle r_{e N}^{6}\right\rangle,\left\langle r_{e e}^{6}\right\rangle,\left\langle r_{e N}^{8}\right\rangle,\left\langle r_{e e}^{8}\right\rangle,\left\langle r_{e N}^{10}\right\rangle$ and $\left\langle r_{e e}^{10}\right\rangle$ expectation values determined to high accuracy in this study can be considered as an important addition to the set of known atomic properties for each of two-electron ions considered. Analogous studies must be performed for other bound states in two-electron ions and atoms, e.g., for 
the $\mathrm{P}-$ and $D$-states. For the $\mathrm{Ps}^{-}$and $\mathrm{H}^{-}$ions the expectation values of the $r_{e N}^{2 k}$ and $r_{e e}^{2 k}$ operators $(k \geq 3)$ are reported for the first time. Accurate $\left\langle r_{e N}^{2 k}\right\rangle$ and $\left\langle r_{e e}^{2 k}\right\rangle$ expectation (for $k=3,4,5, \ldots$ ) are important to investigate the role of electron-electron correlations in few-electron atoms and ions. Based on our accurate results we have developed a modified version of the $Q^{-1}$-expansion for two-electron ions with different nuclear charges $Q$. This method allows one to predict accurate numerical values of the $\left\langle r_{e N}^{6}\right\rangle,\left\langle r_{e e}^{6}\right\rangle,\left\langle r_{e N}^{8}\right\rangle,\left\langle r_{e e}^{8}\right\rangle,\left\langle r_{e N}^{10}\right\rangle$ and $\left\langle r_{e e}^{10}\right\rangle$ expectation values without extensive computations.

[1] N.F. Mott and H.S.W. Massey, Theory of Atomic Collisions, (3rd. ed. Oxford Clarendon Press, Oxford (UK) (1965)).

[2] V.A. Kvlividze and S.S. Krasil'nikov, Physics of Atomic Collisions, (Moscow State University Publ., Moscow (1985)) [in Russian].

[3] H.A. Bethe, Intermediate Quantum Mechanics, (W.A. Benjamin Inc., New York (1964)), Chapt. 15.

[4] L.D. Landau and E.M. Lifshitz, Quantum Mechanics: Non-relativistic Theory, (3rd ed., Pergamonn Press, New York (1976)).

[5] A.I. Akhiezer and V.B. Beresteskii, Quantum Electrodynamics, (4th ed., Science, Moscow (1981)), Chpt. 4 and 5.

[6] W. Greiner and J. Reinhardt, Quantum Electrodynamics, (4th edn., Springer Verlag, Berlin, 2009).

[7] A.M. Frolov, Phys. Rev. E 64, 036704 (2001); ibid, 74, 027702 (2006).

[8] S.T. Epstein, The Variation Method in Quantum Chemistry, (Academic Press, New York, 1974).

[9] A. M. Frolov, Sov. Phys. JETP 92, 1100 (1987) [Zh. Eksp. Teor. Fiz. 92, 1959 (1987)].

[10] A.M. Frolov, Chem. Phys. Lett. 635, 312 (2015) [see also: J. Phys. A 40, 6175 (2007)].

[11] A.M. Frolov, Europ. Phys. Jour. D 69, 132 (2015).

[12] A.M. Frolov, Chem. Phys. Lett. 638108 (2015).

[13] H.A. Bethe and E.E. Salpeter, Quantum Mechanics of One- and Two-Electron Atoms, (Dover Publ. Inc., Mineola, NY, (2008)).

[14] A.K. Bhatia and R.J. Drachman, Phys. Rev. A 28, 2523 (1983). 
[15] Y.K. Ho, Phys. Lett. A 144, 237 (1990).

[16] D.J. Hudson, Lectures on Elementary Statistics and Probability, (MIR, Moscow, 1967), Chpt. VIII [in Russian]. 
TABLE I: Convergence of the expectation values of the 4th and 6th powers of the electron-nuclear and electron-electron distances for the $\mathrm{Ps}^{-}$ion. All values are shown in atomic units and $N$ is the total number of basis functions used.

\begin{tabular}{|c|c|c|}
\hline \hline$N$ & $\left\langle r_{e N}^{4}\right\rangle$ & \multicolumn{1}{|c|}{$\left\langle r_{e e}^{4}\right\rangle$} \\
\hline 700 & $9.93063866354016104309483 \cdot 10^{4}$ & $2.10544533530693363256539 \cdot 10^{4}$ \\
1000 & $9.93063867695759270445863 \cdot 10^{4}$ & $2.10544533816002294010497 \cdot 10^{4}$ \\
1500 & $9.93063867981220599278244 \cdot 10^{4}$ & $2.10544533892937124105159 \cdot 10^{4}$ \\
2000 & $9.93063867979626166786078 \cdot 10^{4}$ & $2.10544533892588984674548 \cdot 10^{4}$ \\
2500 & $9.93063867979600602716265 \cdot 10^{4}$ & $2.10544533892583617688472 \cdot 10^{4}$ \\
3000 & $9.93063867979600416141868 \cdot 10^{4}$ & $2.10544533892583581109197 \cdot 10^{4}$ \\
3500 & $9.93063867979600415981681 \cdot 10^{4}$ & $2.10544533892583581051440 \cdot 10^{4}$ \\
3840 & $9.93063867979600415474280 \cdot 10^{4}$ & $2.10544533892583580951959 \cdot 10^{4}$ \\
\hline \hline$N$ & $\left\langle r_{e N}^{6}\right\rangle$ & $\left\langle r_{e e}^{6}\right\rangle$ \\
\hline 700 & $4.80568068814743249392206 \cdot 10^{6}$ & $9.99929836506863663378819 \cdot 10^{6}$ \\
1000 & $4.80568114031556600853508 \cdot 10^{6}$ & $9.99929927561165508453627 \cdot 10^{6}$ \\
1500 & $4.80568125153088967470141 \cdot 10^{6}$ & $9.99929952003726374529628 \cdot 10^{6}$ \\
2000 & $4.80568125107402346104496 \cdot 10^{6}$ & $9.99929951911244171039228 \cdot 10^{6}$ \\
2500 & $4.80568125106545530348401 \cdot 10^{6}$ & $9.99929951909487275907597 \cdot 10^{6}$ \\
3000 & $4.80568125106541274957314 \cdot 10^{6}$ & $9.99929951909478846572168 \cdot 10^{6}$ \\
3500 & $4.80568125106541138515854 \cdot 10^{6}$ & $9.99929951909478594810657 \cdot 10^{6}$ \\
3840 & $4.80568125106541091239665 \cdot 10^{6}$ & $9.99929951909478493674233 \cdot 10^{6}$ \\
\hline \hline
\end{tabular}


TABLE II: Convergence of the expectation values of 8th and 10th powers of the electron-nuclear and electron-electron distances for the $\mathrm{Ps}^{-}$ion. All values are shown in atomic units and $N$ is the total number of basis functions used.

\begin{tabular}{|c|c|c|}
\hline \hline$N$ & $\left\langle r_{e N}^{8}\right\rangle$ & \multicolumn{1}{|c|}{$\left\langle r_{e e}^{8}\right\rangle$} \\
\hline 700 & $4.24774492689891131699935 \cdot 10^{9}$ & $8.66821845956715753201073 \cdot 10^{9}$ \\
1000 & $4.24772820309251553342332 \cdot 10^{9}$ & $8.66819102432451089731615 \cdot 10^{9}$ \\
2000 & $4.24772576076621174183137 \cdot 10^{9}$ & $8.66818742036339759667927 \cdot 10^{9}$ \\
2500 & $4.24772575007510814637651 \cdot 10^{9}$ & $8.66818739918510066202142 \cdot 10^{9}$ \\
3000 & $4.24772574985746459705903 \cdot 10^{9}$ & $8.66818739874325727683485 \cdot 10^{9}$ \\
3500 & $4.24772574985738081866795 \cdot 10^{9}$ & $8.66818739874058038925848 \cdot 10^{9}$ \\
3840 & $4.24772574985736450997606 \cdot 10^{9}$ & $8.66818739874054267042955 \cdot 10^{9}$ \\
\hline \hline$N$ & $\left\langle r_{e N}^{10}\right\rangle$ & $\left\langle r_{e e}^{10}\right\rangle$ \\
\hline 700 & $6.00429250070626982715951 \cdot 10^{12}$ & $1.2133290535975306506086 \cdot 10^{13}$ \\
1000 & $5.99839628558760769260162 \cdot 10^{12}$ & $1.2124018782302376120116 \cdot 10^{13}$ \\
1500 & $5.99044448718695409309036 \cdot 10^{12}$ & $1.2108720042936564326664 \cdot 10^{13}$ \\
2000 & $5.99044264859592495429707 \cdot 10^{12}$ & $1.2108716381259421804620 \cdot 10^{13}$ \\
2500 & $5.99044264135992752720215 \cdot 10^{12}$ & $1.2108716367982696982857 \cdot 10^{13}$ \\
3000 & $5.99044264126434938468518 \cdot 10^{12}$ & $1.2108716367785339970708 \cdot 10^{13}$ \\
3500 & $5.99044264126106870127531 \cdot 10^{12}$ & $1.2108716367779335976792 \cdot 10^{13}$ \\
3840 & $5.99044264126106995225945 \cdot 10^{12}$ & $1.2108716367779231713846 \cdot 10^{13}$ \\
\hline \hline
\end{tabular}


TABLE III: Convergence of the expectation values of 4th, 6th, 8th and 10th powers of the electronnuclear and electron-electron distances for the $\mathrm{H}^{-}$ion with the infinitely heavy nucleus $\left({ }^{\infty} \mathrm{H}^{-}\right)$. All values are shown in atomic units and $N$ is the total number of basis functions used.

\begin{tabular}{|c|c|c|}
\hline \hline$N$ & $\left\langle r_{e N}^{4}\right\rangle$ & $\left\langle r_{e e}^{4}\right\rangle$ \\
\hline 2500 & $6.45144542412223384151458 \cdot 10^{2}$ & $1.5900946039394936532910 \cdot 10^{3}$ \\
3000 & $6.45144542412219611111303 \cdot 10^{2}$ & $1.5900946039394858104929 \cdot 10^{3}$ \\
3500 & $6.45144542412219389586010 \cdot 10^{2}$ & $1.5900946039394853349180 \cdot 10^{3}$ \\
4000 & $6.45144542412219370980781 \cdot 10^{2}$ & $1.5900946039394852936401 \cdot 10^{3}$ \\
\hline \hline$N$ & $\left\langle r_{e N}^{6}\right\rangle$ & $\left\langle r_{e e}^{6}\right\rangle$ \\
\hline 2500 & $8.72661424069925625718951 \cdot 10^{4}$ & $2.1253344237087617700834 \cdot 10^{5}$ \\
3000 & $8.72661424069612058896071 \cdot 10^{4}$ & $2.1253344237081430766096 \cdot 10^{5}$ \\
3500 & $8.72661424069594537727044 \cdot 10^{4}$ & $2.1253344237081083393021 \cdot 10^{5}$ \\
4000 & $8.72661424069592696935431 \cdot 10^{4}$ & $2.1253344237081047127748 \cdot 10^{5}$ \\
\hline \hline$N$ & $\left\langle r_{e N}^{8}\right\rangle$ & $\left\langle r_{e e}^{8}\right\rangle$ \\
\hline 2500 & $2.2035718695630030404609 \cdot 10^{7}$ & $5.2218676447807289690830 \cdot 10^{7}$ \\
3000 & $2.2035718695497230895897 \cdot 10^{7}$ & $5.2218676447555114704017 \cdot 10^{7}$ \\
3500 & $2.2035718695491884707056 \cdot 10^{7}$ & $5.2218676447545607058237 \cdot 10^{7}$ \\
4000 & $2.2035718695491376572405 \cdot 10^{7}$ & $5.2218676447544884570831 \cdot 10^{7}$ \\
\hline \hline$N$ & $\left\langle r_{e N}^{10}\right\rangle$ & $\left\langle r_{e e}^{10}\right\rangle$ \\
\hline 2500 & $8.9379707582741862889150 \cdot 10^{9}$ & $2.0815154470686552817302 \cdot 10^{10}$ \\
3000 & $8.9379707583541128919721 \cdot 10^{9}$ & $2.0815154470904085768025 \cdot 10^{10}$ \\
3500 & $8.9379707584098506889807 \cdot 10^{9}$ & $2.0815154471021717913050 \cdot 10^{10}$ \\
4000 & $8.9379707584209025581073 \cdot 10^{9}$ & $2.0815154471047399049048 \cdot 10^{10}$ \\
\hline \hline
\end{tabular}


TABLE IV: Expectation values of the $r_{e N}^{k}$ operators for $k=6,8$ and 10 (in a.u.) for the ground $1^{1} S$-state(s) in some two-electron atoms and ions.

\begin{tabular}{|c|c|c|c|}
\hline ion & $\left\langle r_{e N}^{6}\right\rangle$ & $\left\langle r_{e N}^{8}\right\rangle$ & $\left\langle r_{e N}^{10}\right\rangle$ \\
\hline $\mathrm{H}^{-}$ & $8.7266142406959270 \cdot 10^{6}$ & $2.20357186954914 \cdot 10^{7}$ & $8.9379707584209 \cdot 10^{9}$ \\
\hline $\mathrm{He}$ & 26.282446975525706 & 289.827674593716 & 4797.7265440809 \\
\hline $\mathrm{Li}^{+}$ & 1.2153694626387516 & 4.57848899403444 & 25.643305753941 \\
\hline $\mathrm{Be}^{2+}$ & 0.16289999058428645 & 0.307966596103007 & 0.86217163108619 \\
\hline $\mathrm{B}^{3+}$ & $3.6352298112380881 \cdot 10^{-2}$ & $4.12490989522882 \cdot 10^{-2}$ & $6.9160629356510 \cdot 10^{-2}$ \\
\hline $\mathrm{C}^{4+}$ & $1.0971961307603750 \cdot 10^{-2}$ & $8.29648580341205 \cdot 10^{-3}$ & $9.2569896501927 \cdot 10^{-3}$ \\
\hline $\mathrm{N}^{5+}$ & $4.0458545441354921 \cdot 10^{-3}$ & $2.18393437236692 \cdot 10^{-3}$ & $1.7378981971464 \cdot 10^{-3}$ \\
\hline $\mathrm{O}^{6+}$ & $1.7207164884993150 \cdot 10^{-3}$ & $6.96222590624758 \cdot 10^{-4}$ & $4.1499485730461 \cdot 10^{-4}$ \\
\hline $\mathrm{F}^{7+}$ & $8.1438807613848554 \cdot 10^{-4}$ & $2.56150307414319 \cdot 10^{-4}$ & $1.1862759922226 \cdot 10^{-4}$ \\
\hline $\mathrm{Ne}^{8+}$ & $4.1882979208164487 \cdot 10^{-4}$ & $1.05338028889520 \cdot 10^{-4}$ & $3.8992426943743 \cdot 10^{-5}$ \\
\hline $\mathrm{Na}^{9+}$ & $2.3019815537090110 \cdot 10^{-4}$ & $4.73497099763261 \cdot 10^{-5}$ & $1.4329626527333 \cdot 10^{-5}$ \\
\hline $\mathrm{Mg}^{10+}$ & $1.3358999138069766 \cdot 10^{-4}$ & $2.28900874982039 \cdot 10^{-5}$ & $5.7690341921676 \cdot 10^{-6}$ \\
\hline $\mathrm{Al}^{11+}$ & $8.1118206099732403 \cdot 10^{-5}$ & $1.17570648695508 \cdot 10^{-5}$ & $2.5058841914728 \cdot 10^{-6}$ \\
\hline $\mathrm{Si}^{12+}$ & $5.1181008635663217 \cdot 10^{-5}$ & $6.35647216973809 \cdot 10^{-6}$ & $1.1606982837876 \cdot 10^{-6}$ \\
\hline $\mathrm{Ph}^{13+}$ & $3.3370916846780790 \cdot 10^{-5}$ & $3.59099339836029 \cdot 10^{-6}$ & $5.6804564641147 \cdot 10^{-6}$ \\
\hline $\mathrm{S}^{14+}$ & $2.2386933069629092 \cdot 10^{-5}$ & $2.10740403639127 \cdot 10^{-6}$ & $2.9158091284563 \cdot 10^{-7}$ \\
\hline $\mathrm{Cl}^{15+}$ & $1.5397251950625338 \cdot 10^{-5}$ & $1.27863754695153 \cdot 10^{-6}$ & $1.5604637828244 \cdot 10^{-7}$ \\
\hline $\operatorname{Ar}^{16+}$ & $1.0825341985580918 \cdot 10^{-5}$ & $7.98933602270988 \cdot 10^{-7}$ & $8.6642831006576 \cdot 10^{-8}$ \\
\hline $\mathrm{K}^{17+}$ & $7.7612111364041836 \cdot 10^{-6}$ & $5.12411362175347 \cdot 10^{-7}$ & $4.9707090637754 \cdot 10^{-8}$ \\
\hline $\mathrm{Ca}^{18+}$ & $5.6625739359113182 \cdot 10^{-6}$ & $3.36416344886887 \cdot 10^{-7}$ & $2.9364007261172 \cdot 10^{-8}$ \\
\hline $\mathrm{Sc}^{19+}$ & $4.1969687507078452 \cdot 10^{-6}$ & $2.25564343790269 \cdot 10^{-7}$ & $1.7809620119723 \cdot 10^{-8}$ \\
\hline $\mathrm{Ti}^{20+}$ & $3.1553271100240321 \cdot 10^{-6}$ & $1.54144738758072 \cdot 10^{-7}$ & $1.1062488796844 \cdot 10^{-8}$ \\
\hline $\mathrm{V}^{21+}$ & $2.4031333585703164 \cdot 10^{-6}$ & $1.07176714358115 \cdot 10^{-7}$ & $7.0223414061394 \cdot 10^{-9}$ \\
\hline $\mathrm{Cr}^{22+}$ & $1.8520276933457352 \cdot 10^{-6}$ & $7.57063449688798 \cdot 10^{-8}$ & $4.5472539112903 \cdot 10^{-8}$ \\
\hline $\mathrm{Mn}^{23+}$ & $1.4428645036187975 \cdot 10^{-6}$ & $5.42565975946396 \cdot 10^{-8}$ & $2.9990536557991 \cdot 10^{-9}$ \\
\hline $\mathrm{Fe}^{24+}$ & $1.1353655907960862 \cdot 10^{-6}$ & $3.94056247079358 \cdot 10^{-8}$ & $2.0120181986675 \cdot 10^{-9}$ \\
\hline $\mathrm{Co}^{25+}$ & $9.0166466631135737 \cdot 10^{-7}$ & $2.89737207550905 \cdot 10^{-8}$ & $1.3717427143265 \cdot 10^{-9}$ \\
\hline $\mathrm{Ni}^{26+}$ & $7.2220007860909793 \cdot 10^{-7}$ & $2.15474156619540 \cdot 10^{-8}$ & $9.5023197194145 \cdot 10^{-10}$ \\
\hline
\end{tabular}


TABLE V: Expectation values of the $r_{e e}^{k}$ operators for $k=6,8$ and 10 (in a.u.) for the ground $1^{1} S$-state(s) in some two-electron atoms and ions.

\begin{tabular}{|c|c|c|c|}
\hline ion & $\left\langle r_{e e}^{6}\right\rangle$ & $\left\langle r_{e e}^{8}\right\rangle$ & $\left\langle r_{e e}^{10}\right\rangle$ \\
\hline $\mathrm{H}^{-}$ & $2.1253344237081047 \cdot 10^{5}$ & $5.221867644754489 \cdot 10^{7}$ & $8.9379707584209 \cdot 10^{10}$ \\
\hline $\mathrm{He}$ & $1.1245631448121300 \cdot 10^{2}$ & $1.470413341085919 \cdot 10^{3}$ & $2.7119255221934 \cdot 10^{4}$ \\
\hline $\mathrm{Li}^{+}$ & 5.6292546484902171 & 26.91030017485420 & 180.01353891601 \\
\hline $\mathrm{Be}^{2+}$ & $7.7576731467508007 \cdot 10^{-1}$ & 1.915698537380722 & 6.6096105596883 \\
\hline $\mathrm{B}^{3+}$ & $1.7548284756742420 \cdot 10^{-1}$ & $2.642503536372184 \cdot 10^{-1}$ & $5.5571366091173 \cdot 10^{-1}$ \\
\hline $\mathrm{C}^{4+}$ & $5.3383732025165301 \cdot 10^{-2}$ & $5.410677232398524 \cdot 10^{-2}$ & $7.6573683711407 \cdot 10^{-2}$ \\
\hline $\mathrm{N}^{5+}$ & $1.9785477877402386 \cdot 10^{-2}$ & $1.441400250285469 \cdot 10^{-2}$ & $1.4661687535537 \cdot 10^{-2}$ \\
\hline $\mathrm{O}^{6+}$ & $8.4446682997834888 \cdot 10^{-3}$ & $4.634451576888273 \cdot 10^{-3}$ & $3.5511567156249 \cdot 10^{-3}$ \\
\hline $\mathrm{F}^{7+}$ & $4.0071008219829092 \cdot 10^{-3}$ & $1.716011552310627 \cdot 10^{-3}$ & $1.0260492148249 \cdot 10^{-3}$ \\
\hline $\mathrm{Ne}^{8+}$ & $2.0648758933294060 \cdot 10^{-3}$ & $7.091935926647552 \cdot 10^{-4}$ & $3.4009437094400 \cdot 10^{-4}$ \\
\hline $\mathrm{Na}^{9+}$ & $1.1366636836485451 \cdot 10^{-3}$ & $3.200492444138807 \cdot 10^{-4}$ & $1.2582662493278 \cdot 10^{-4}$ \\
\hline $\mathrm{Mg}^{10+}$ & $6.6046023981302165 \cdot 10^{-4}$ & $1.552212566637046 \cdot 10^{-4}$ & $5.0937094007657 \cdot 10^{-5}$ \\
\hline $\mathrm{Al}^{11+}$ & $4.0145512771582814 \cdot 10^{-4}$ & $7.994117953375549 \cdot 10^{-5}$ & $2.2227468708512 \cdot 10^{-5}$ \\
\hline $\mathrm{Si}^{12+}$ & $2.5351297160481673 \cdot 10^{-4}$ & $4.331857470689201 \cdot 10^{-5}$ & $1.0335731248035 \cdot 10^{-5}$ \\
\hline $\mathrm{Ph}^{13+}$ & $1.6541519866000751 \cdot 10^{-4}$ & $2.451978179637930 \cdot 10^{-5}$ & $5.0752523190550 \cdot 10^{-6}$ \\
\hline $\mathrm{S}^{14+}$ & $1.1103834367892564 \cdot 10^{-4}$ & $1.441385237818165 \cdot 10^{-5}$ & $2.6127229815422 \cdot 10^{-6}$ \\
\hline $\mathrm{Cl}^{15+}$ & $7.6411142361518097 \cdot 10^{-5}$ & $8.758258104512842 \cdot 10^{-6}$ & $1.4018157968702 \cdot 10^{-6}$ \\
\hline $\operatorname{Ar}^{16+}$ & $5.3747821872842266 \cdot 10^{-5}$ & $5.479528022775409 \cdot 10^{-6}$ & $7.8008737661896 \cdot 10^{-7}$ \\
\hline $\mathrm{K}^{17+}$ & $3.8550525434837112 \cdot 10^{-5}$ & $3.518441099086186 \cdot 10^{-6}$ & $4.4842890525060 \cdot 10^{-7}$ \\
\hline $\mathrm{Ca}^{18+}$ & $2.8136886884911498 \cdot 10^{-5}$ & $2.312355602765375 \cdot 10^{-6}$ & $2.6537638458779 \cdot 10^{-7}$ \\
\hline $\mathrm{Sc}^{19+}$ & $2.0861340806322627 \cdot 10^{-5}$ & $1.551847129149528 \cdot 10^{-6}$ & $1.6120980250305 \cdot 10^{-7}$ \\
\hline $\mathrm{Ti}^{20+}$ & $1.5688474182661537 \cdot 10^{-5}$ & $1.061376785918574 \cdot 10^{-6}$ & $1.0027749296477 \cdot 10^{-7}$ \\
\hline $\mathrm{V}^{21+}$ & $1.1951752839024337 \cdot 10^{-5}$ & $7.385339159464128 \cdot 10^{-7}$ & $6.3733691984626 \cdot 10^{-8}$ \\
\hline $\mathrm{Cr}^{22+}$ & $9.2131446340301449 \cdot 10^{-6}$ & $5.220384819335957 \cdot 10^{-7}$ & $4.1312674172434 \cdot 10^{-8}$ \\
\hline $\mathrm{Mn}^{23+}$ & $7.1793199944813362 \cdot 10^{-6}$ & $3.743671833575214 \cdot 10^{-7}$ & $2.7267628879708 \cdot 10^{-8}$ \\
\hline $\mathrm{Fe}^{24+}$ & $5.6504452814749105 \cdot 10^{-6}$ & $2.720546821859721 \cdot 10^{-7}$ & $1.8300150240156 \cdot 10^{-8}$ \\
\hline $\mathrm{Co}^{25+}$ & $4.4882185523678996 \cdot 10^{-6}$ & $2.001406932108432 \cdot 10^{-7}$ & $1.2473265480770 \cdot 10^{-8}$ \\
\hline $\mathrm{Ni}^{26+}$ & $3.5955234289593961 \cdot 10^{-6}$ & $1.489162219104389 \cdot 10^{-7}$ & $8.6255426406327 \cdot 10^{-9}$ \\
\hline
\end{tabular}


TABLE VI: Convergence of the $\left\langle r_{e N}^{6}\right\rangle$ and $\left\langle r_{e N}^{8}\right\rangle$ expectation values for the two-electron $\mathrm{K}^{17+}$ ion determined with the use of the $M$-terms in the $Q^{-1}$-expansion, Eq.(11).

\begin{tabular}{|c|c|c|}
\hline \hline$M$ & $\left\langle r_{e N}^{6}\right\rangle$ & $\left\langle r_{e N}^{8}\right\rangle$ \\
\hline 14 & $7.76122879758595388 \mathrm{E}-06$ & $5.12412920398821052 \mathrm{E}-07$ \\
16 & $7.76121135024542466 \mathrm{E}-06$ & $5.12411380495447621 \mathrm{E}-07$ \\
18 & $7.76121108013487460 \mathrm{E}-06$ & $5.12411356758053888 \mathrm{E}-07$ \\
20 & $7.76121113844470425 \mathrm{E}-06$ & $5.12411362696037344 \mathrm{E}-07$ \\
22 & $7.76121113636655254 \mathrm{E}-06$ & $5.12411362053147883 \mathrm{E}-07$ \\
24 & $7.76121113640500495 \mathrm{E}-06$ & $5.12411362204730508 \mathrm{E}-07$ \\
\hline 'exact' ${ }^{\prime(a)}$ & $7.7612111364041836 \cdot 10^{-6}$ & $5.12411362175347 \cdot 10^{-7}$ \\
\hline \hline
\end{tabular}

${ }^{(a)}$ See Table IV. 